University of Wollongong

Research Online

Faculty of Engineering and Information

Faculty of Engineering and Information

Sciences - Papers: Part B

Sciences

2018

\title{
Effect of polarization sensitivity on ultrasmall silicon-on-insulator-based arrayed waveguide grating for fiber Bragg grating sensor interrogation
}

Hongqiang Li

Tianjin Polytechnic University, lihongqiang@tjpu.edu.cn

Rui Xie

Tianjin Polytechnic University

Yanhua Hong

Bangor University

Zanyun Zhang

Tianjin Polytechnic University

Cheng Zhang

Tianjin Polytechnic University

See next page for additional authors

Follow this and additional works at: https://ro.uow.edu.au/eispapers1

Part of the Engineering Commons, and the Science and Technology Studies Commons

Research Online is the open access institutional repository for the University of Wollongong. For further information contact the UOW Library: research-pubs@uow.edu.au 


\title{
Effect of polarization sensitivity on ultrasmall silicon-on-insulator-based arrayed waveguide grating for fiber Bragg grating sensor interrogation
}

\author{
Abstract \\ Polarization sensitivity is an important factor that affects the interrogation of ultrasmall arrayed \\ waveguide grating (AWG) for fiber Bragg grating (FBG) sensor. An ultrasmall $1 \times 8$ silicon-on-insulator \\ (Sol) AWG with a core size of less than $530 \mu \mathrm{m} \times 480 \mu \mathrm{m}$ is proposed in this study. This ultrasmall Sol \\ AWG exhibits good transmission spectra and high polarization sensitivity. The increased channel \\ numbers and tight structure increase the polarization sensitivity of AWG. Temperature interrogation \\ experiments show that the FBG sensor interrogation is drastically affected when the effect of polarization \\ sensitivity on the ultrasmall AWG is sufficiently large. \\ Disciplines \\ Engineering | Science and Technology Studies

\section{Publication Details} \\ Li, H., Xie, R., Hong, Y., Zhang, Z., Zhang, C., Tang, C. \& Li, E. (2018). Effect of polarization sensitivity on \\ ultrasmall silicon-on-insulator-based arrayed waveguide grating for fiber Bragg grating sensor \\ interrogation. Optical Engineering, 57 (6), 065103-1-065103-7.

\section{Authors} \\ Hongqiang Li, Rui Xie, Yanhua Hong, Zanyun Zhang, Cheng Zhang, Chunxiao Tang, and Enbang Li
}




\title{
Effect of polarization sensitivity on ultrasmall silicon-on-insulator-based arrayed waveguide grating for fiber Bragg grating sensor interrogation
}

\author{
Hongqiang Li, ${ }^{a}$ 用 Rui Xie, ${ }^{a}$ Yanhua Hong, ${ }^{b}$ Zanyun Zhang, ${ }^{a}$ Cheng Zhang, ${ }^{a}$ Chunxiao Tang, ${ }^{a}$ and Enbang Lic \\ ${ }^{a}$ Tianjin Polytechnic University, School of Electronics and Information Engineering, Tianjin Key Laboratory of Optoelectronic Detection Technology \\ and Systems, Tianjin, China

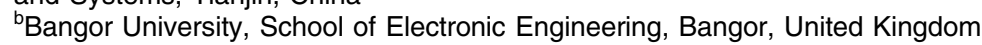 \\ 'University of Wollongong, School of Physics, Wollongong, Australia
}

\begin{abstract}
Polarization sensitivity is an important factor that affects the interrogation of ultrasmall arrayed waveguide grating (AWG) for fiber Bragg grating (FBG) sensor. An ultrasmall $1 \times 8$ silicon-on-insulator (Sol) AWG with a core size of less than $530 \mu \mathrm{m} \times 480 \mu \mathrm{m}$ is proposed in this study. This ultrasmall Sol AWG exhibits good transmission spectra and high polarization sensitivity. The increased channel numbers and tight structure increase the polarization sensitivity of AWG. Temperature interrogation experiments show that the FBG sensor interrogation is drastically affected when the effect of polarization sensitivity on the ultrasmall AWG is sufficiently

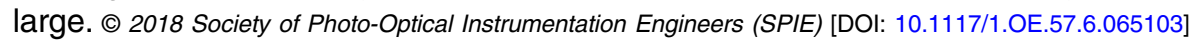

Keywords: arrayed waveguide grating; polarization; silicon-on-insulator; fiber Bragg grating.

Paper 180357 received Mar. 6, 2018; accepted for publication Jun. 5, 2018; published online Jun. 27, 2018.

\section{Introduction}

Fiber Bragg grating (FBG) sensors have attracted increasing attention, because they provide considerable advantages over conventional electrical sensors 90 Several FBG sensor interrogation methods - such as dynamic matched grating filtering, ,Fabry-Perot filter method, Mach-Zehnder modulator method, 102 tunable narrow-line width laser scanning, and arrayed waveguide grating (AWG) interrogation-14 [15 have been proposed. Among these FBG sensor interrogation methods, the dynamic matched grating filtering method exhibits the simplest structure and the lowest accuracy. The Fabry-Perot filter method possesses high resolution but requires complicated equipment. The Mach-Zehnder method cannot be used in quasistatic measurements. The tunable narrow-line width laser scanning method requires high amounts of light. The AWG-based FBG sensor interrogation system has attracted attention because of its high precision and high speed. However, traditional AWGs cannot satisfy the latest requirements for miniaturization given their large sizes. Thus, a specially designed AWG with suitable size and performance for AWG interrogation must be developed.

A compact AWG can be fabricated on a silicon-on-insulator (SoI)-based platform because of its ultrahigh relative refractive index difference in the Si core and low-index claddings. These characteristics allow for sharp bends and enable size reduction to the micrometer, as well as the integration of scaled-up structures with other devices in a microsystem. Therefore, SoI-based AWG miniaturization warrants further study. Yang et al $\$$ proposed a $48 \times 48$ AWG on an SoI platform with $0.8-\mathrm{nm}$ channel spacing. The fabricated AWG has a compact size of approximately $220 \mu \mathrm{m} \times 470 \mu \mathrm{m}$ and

*Address all correspondence to: Hongqiang Li, E-mail: thongqiang@tjpu.edu $\mathrm{cn}$ exhibits an insertion loss and a crosstalk of less than -4 and $-15 \mathrm{~dB}$, respectively. Wang et al 18 proposed an $8 \times 8$ silicon nanowire AWG that has a crosstalk of approximately $-17 \mathrm{~dB}$, an insertion loss of approximately $-2.92 \mathrm{~dB}$, and a footprint of only $730 \mu \mathrm{m} \times 300 \mu \mathrm{m}$. Park et al 19 fabricated an eight-channel AWG that demonstrates an insertion loss of $0.63 \mathrm{~dB}$ and a crosstalk of -23 to $-25.3 \mathrm{~dB}$. Zou et al. ${ }^{0}$ fabricated two SoI AWG routers that exhibit significantly improved spectral responses relative to those exhibited by SoI AWG routers with conventional design. However, the effect of polarization sensitivity on ultrasmall SoI AWG for an FBG sensor interrogation system remains uninvestigated.

This study proposes a $1 \times 8$ SoI AWG, which possess core sizes of $350 \mu \mathrm{m} \times 250 \mu \mathrm{m}$. Simulation results show that these ultrasmall SoI AWGs exhibit large wavelength drift under transverse electric (TE) and transverse magnetic (TM) modes. The polarization sensitivity of the AWG increases because of the increase in channel number and tight structure. The experimental results show that the $1 \times 8$ AWG has an insertion loss of less than $-3.4 \mathrm{~dB}$, a crosstalk of less than $-23.4 \mathrm{~dB}$, and a channel spacing of $1.9 \mathrm{~nm}$. Temperature interrogation experiments show that the proposed FBG sensor interrogation system that is based on the $1 \times 8$ AWG displays a high-interrogation accuracy over the range of $10^{\circ} \mathrm{C}$ to $50^{\circ} \mathrm{C}$ and temperature measurement errors of less than $0.3^{\circ} \mathrm{C}$.

\section{Principle, Design, and Analysis}

The proposed FBG sensor interrogation system consists of a light source, a $2 \times 2$ multimode interference coupler, $1 \times N / 2$ FBG distributed sensors, a $1 \times N$ SoI AWG, ${ }^{23}$ a $1 \times N$ photodetector array, ${ }^{3}$ subsequent signal amplification circuits, an analog-to-digital converter, and a data processing

$0091-3286 / 2018 / \$ 25.00$ (C) 2018 SPIE 


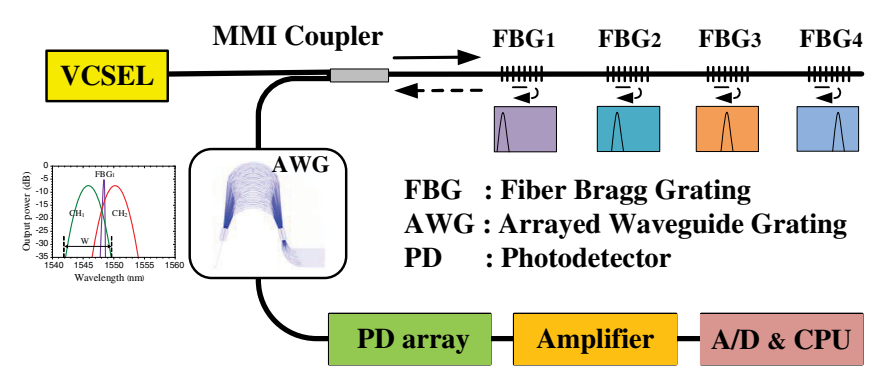

(a)

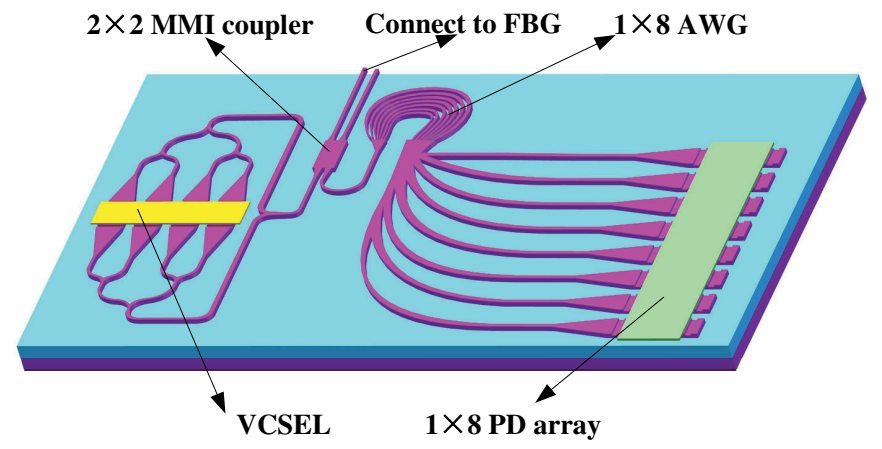

(b)

Fig. 1 Schematic of the proposed FBG sensor interrogation system: (a) schematic of the AWG interrogation of FBG sensors and (b) hybrid Si photonic chip for the proposed FBG sensor interrogator system.

unit [Fig. 1(a)]. The light from the light source penetrates the FBG through the coupler. The reflected light also penetrates the AWG through the coupler. In case of interference from other AWG channels, the central wavelength of each FBG must be in the middle of the central wavelengths of two adjacent AWG channels. When the related variables that affect the spectrum of FBG change, the spectrum will shift and its overlap changes with each AWG channel, thus changing the light intensity in each channel. Each adjacent AWG channel can be used to demodulate variables. SoI is a well-known microelectronics and optoelectronics platform. Extremely small devices can be fabricated on SoI substrates because of the ultrahigh refractive index between $\mathrm{Si}$ and $\mathrm{SiO}_{2}$. A schematic of the hybrid silicon photonic chip for the proposed FBG sensor interrogator system is shown in Fig. 1(b).

The $1 \times N$ AWG is the key component of the FBG sensor interrogation system. In this study, three types of ultrasmall $1 \times N$ SoI AWGs are designed. These AWGs consist of input, slab, array, and output waveguides, as shown in Fig. 2(a). Figure 2(b) shows the structure of the spot-size converter. Light is launched into the planar waveguide through the input spot-size converter. The planar waveguide is designed in accordance with the Rowland circle principle, wherein it can diffract light in the arrayed waveguide. Each arrayed waveguide is located on a circle with its center located at the end of the center input waveguide. The diffracted light enters the arrayed waveguides in the same phase. Figure 2(c) presents the cross-sectional schematic of the arrayed waveguides of the AWG. In the arrayed waveguides, a length difference $(\Delta L)$ is observed between adjacent waveguides. Thus, lights of different wavelengths can attain the same phase difference in the waveguide output. This phase difference results in wavelength-dependent wavefront tilting. The lights with different wavelengths will then focus on each output spot-size converter. Finally, the results of all output gratings are measured by a spectrometer, and then a spectrogram is plotted. This spectrogram can be used to analyze the different types of AWG problems.

When light is transmitted in AWG, the different polarization states of the frequencies of the TE and TM modes shift because waveguide birefringence directly affects the performances of the AWG devices. $n_{\mathrm{TE}}$ and $n_{\mathrm{TM}}$ are assumed to be the TE and TM modes of the effective index rate, respectively. The waveguide birefringence is thus $B=n_{\mathrm{TE}}-n_{\mathrm{TM}}$. The wavelength shift attributed to TE and TM modes is assumed to be $\Delta \lambda$. The center wavelength that corresponds to the polarization state is expressed as follows:

$$
\begin{aligned}
& \lambda_{\mathrm{TM}}(f)=c / f n_{\mathrm{TM}}(f), \\
& \lambda_{\mathrm{TE}}(f)=c / f n_{\mathrm{TE}}(f),
\end{aligned}
$$

where $f$ is the center frequency and $c$ is the light velocity. $\Delta \lambda$ is expressed as follows:

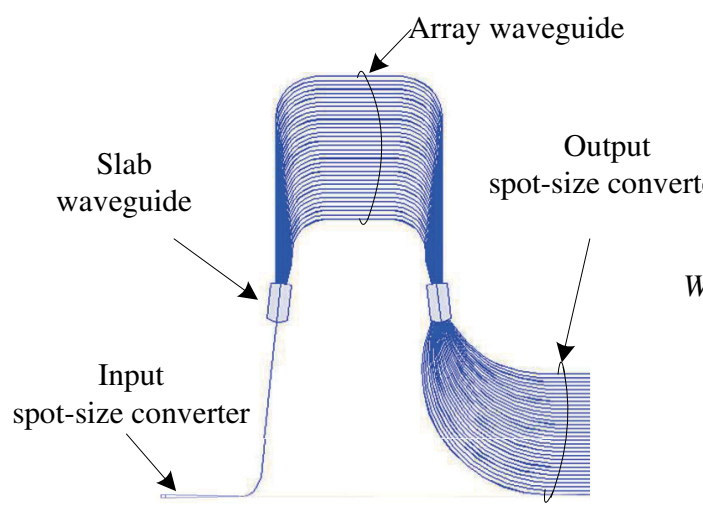

(a)

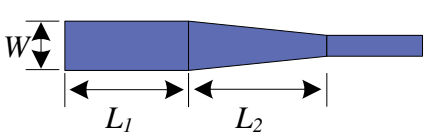

Spot-size converter

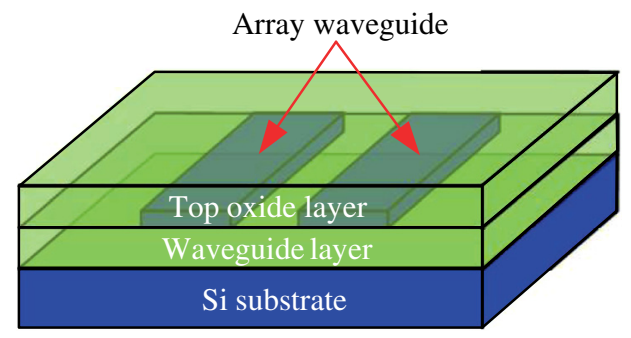

(c)

Fig. 2 (a) AWG principle, (b) structure of the spot-size converter, and (c) cross-sectional schematics of the arrayed waveguides of the AWG. 
$\Delta \lambda=c B / f n_{\mathrm{TE}} n_{\mathrm{TM}}$,

where $W$ is the channel width of the $1 \times N$ AWGs. The increment in the channel width will affect the interrogation results because of the polarization effect. The interrogation results will be unaffected when the wavelength drift is less than the wavelength spacing of the AWG but will be seriously affected when $\Delta \lambda$ is large. The output wavelength shift of the AWG under TE and TM modes is shown in Fig. 3(a). Figure $3(\mathrm{~b})$ shows the schematic of the FBG-reflected wave in the measurement results of the $1 \times N$ AWG. When the channel width of the AWG is considerably large, a large channel crosstalk will occur, causing the reflected signals of the FBG to enter numerous channels. Thus, the temperature interrogation system will not work properly.

Different types of spot-size converters and AWGs are designed through the finite-difference time-domain (FDTD) method. The OptiFDTD simulation software is used for parameter optimization in the simulation experiment. The results show that the AWG with a $0.35 \mu \mathrm{m}$ waveguide width exhibits the best transmission spectrum. Figure 4 presents the transmission efficiency of the input spot-size converter with different parameters. Figure 4(a) depicts that the change in $W$ minimally affects transmission efficiency. Moreover, the change in $W$ negligibly affects transmission efficiency when $L_{2}$ is greater than $90 \mu \mathrm{m}$ [Fig. 4(b)] Under such results, the parameters of the input spot-size converter can be set as $W=6 \mu \mathrm{m}$ and $L_{2}=95 \mu \mathrm{m}$.

The simulation results of the input spot-size converter are presented in Fig. 5(a), which shows that the insertion loss of the input spot-size converters is approximately less than $-0.6 \mathrm{~dB}$. The same method is used to study the output spot-size converter. The simulation results of the output spot-size converter are presented in Fig. 5(b), which shows that the insertion loss of the output spot-size converters is approximately less than $-1.3 \mathrm{~dB}$.

In this study, the AWG structure is optimized by designing different waveguide widths and shapes to obtain the parameters for a high-performance AWG. A $1 \times 8$ AWG is designed with 30 arrayed waveguides and the core size of $350 \mu \mathrm{m} \times 250 \mu \mathrm{m}$. The arrayed waveguide width of all the AWGs is $0.35 \mu \mathrm{m}$. The simulation results show that the $1 \times 8$ AWG with 30 arrayed waveguides exhibits the best transmission spectra, with an insertion loss of less than $-3.2 \mathrm{~dB}$ and a crosstalk of less than $-23.7 \mathrm{~dB}$, as shown in Fig. 6 .

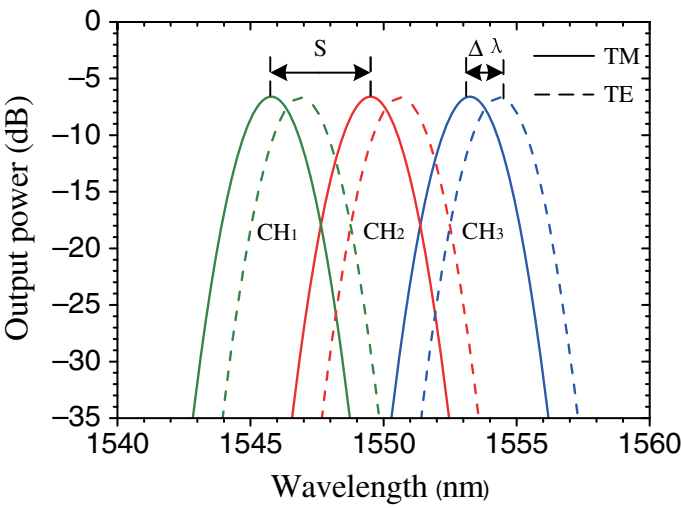

(a)

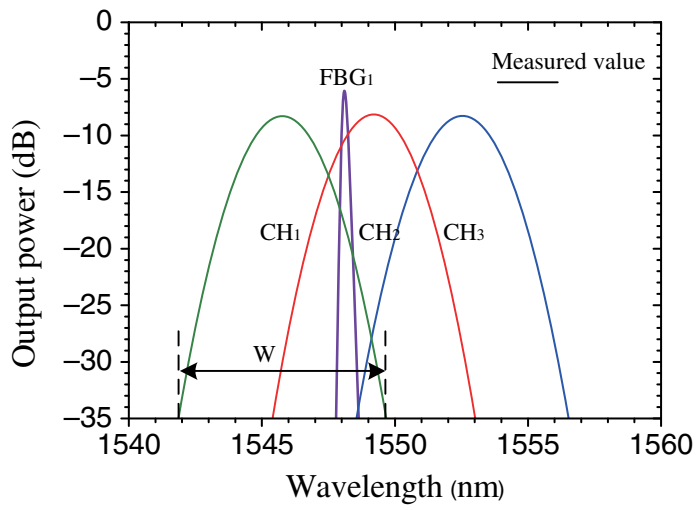

(b)

Fig. 3 (a) Output wavelength shift diagram of AWG under TE and TM modes and (b) schematic of FBG-reflected wave in the measurement results of the $1 \times N$ AWG.

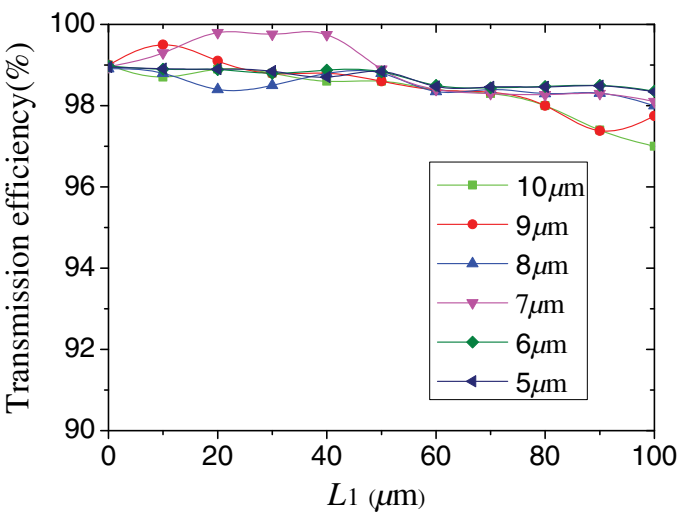

(a)

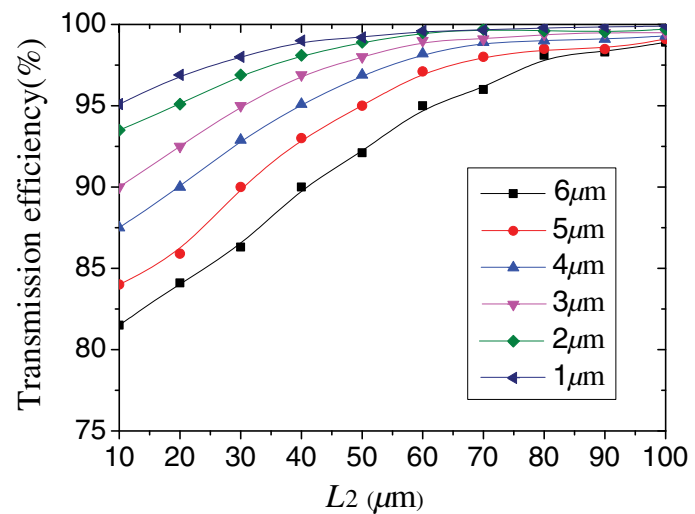

(b)

Fig. 4 Simulation results for input spot-size converter with different parameters. (a) Transmission efficiency as a function of the input spot-size converter length of $L_{2}$ with different $L_{1}$ values. (b) Transmission efficiency as a function of the input spot-size converter length of $L_{2}$ with different $W \mathrm{~s}$. 

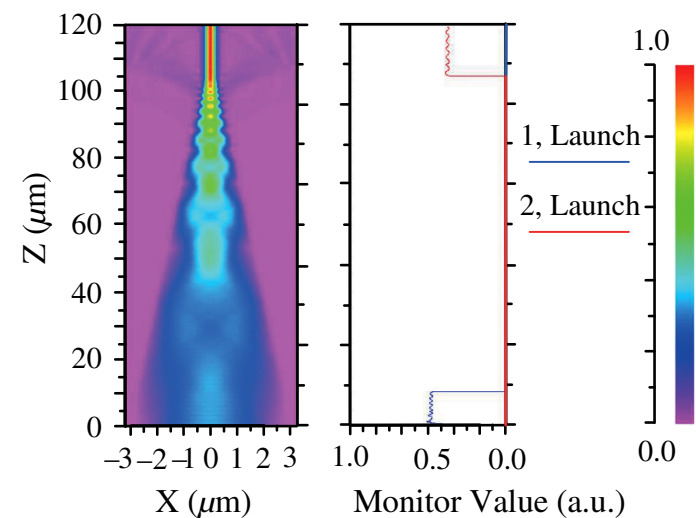

(a)

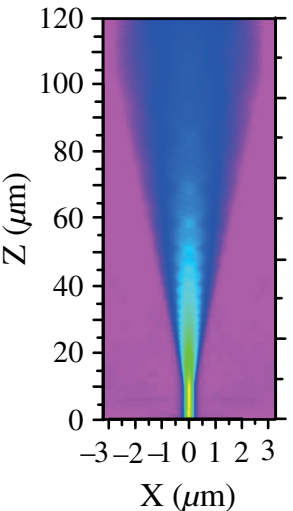

$\mathrm{X}(\mu \mathrm{m})$

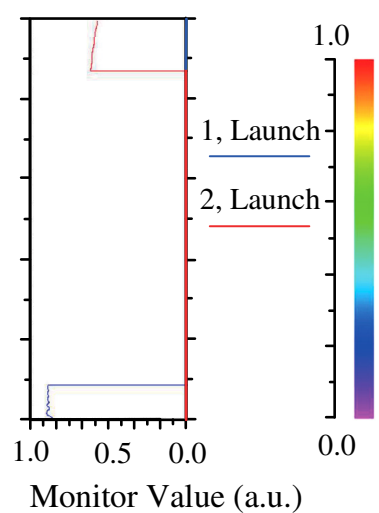

(b)

Fig. 5 Simulation results of (a) input and (b) output spot-size converters: 1, Launch and 2, Launch represent the input light power of the spot-size converter, respectively.

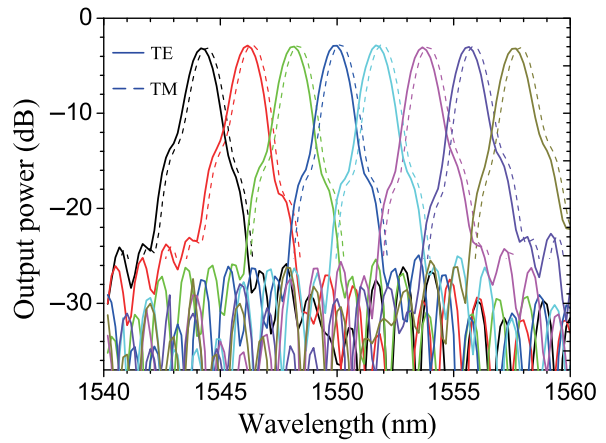

Fig. 6 Simulation results of the $1 \times 8$ AWG with 30 arrayed waveguides.

The simulation results of the three types of AWGs are presented in Table 1, which shows that the channel spacing and the wavelength drift of the $1 \times 8$ AWG are 1.9 and $0.3 \mathrm{~nm}$, respectively. This wavelength drift is small relative to the wavelength spacing, thus implying that the $1 \times 8$ AWG displays high-resolution accuracy in temperature interrogation experiments.

\section{Experimental Results of the Optical Elements}

In reference to the comprehensively optimized design shown above, the designed AWGs and their photonic integrated system are fabricated on a standard SoI wafer with a 220-nmthick top Si and a $2-\mu$ m-thick buried oxide. The AWGs are fabricated at the Institute of Microelectronics, Singapore, via electron beam exposure and response-coupled plasma technology. The microscopy images of the local parts of the spot-size converter are shown in Figs. 7(a) and 7(b). All of the AWGs have the same input and output spot-size converters. Figures [(c) shows the microscopy images of the $1 \times 8$ AWG.
The experiment is performed by using the optical experimental setup shown in Fig. 8(a). The optical experimental setup includes a light source (10-mW SLED, 1550-nm wavelength), tapered lensed single-mode fibers (2-m long, 900- $\mu \mathrm{m}$ Hytrel loose-tube buffered fibre, 7-mm stripped length, $2 \pm 0.5 \mu \mathrm{m}$ spot diameter, and $12 \pm 3 \mu \mathrm{m}$ working distance), a fiber-waveguide coupling alignment system ( $\pm 0.1 \mathrm{~dB}$ alignment accuracy, $\pm 0.1 \mathrm{~dB} / \mathrm{h}$ stability, $\leq 0.5 \mu \mathrm{m}$ gap accuracy, and 1 time W.D. $68 \mathrm{~mm}$ LED light), and a spectrum analyzer $(-70 \mathrm{dBm}$ sensitivity and 0.050 -nm resolution). The tapered lensed single-mode fibers are used to connect the SoI devices and spectrum analyzer. The fiberwaveguide coupling alignment system is used to align the optical fiber and $\mathrm{Si}$ waveguide. The experimental results show that the insertion loss of the input spot-size converter is $-0.9 \mathrm{~dB}$, whereas that of the output spot-size converter is $-1.7 \mathrm{~dB}$. The input and output waveguide width of the AWG is $0.35 \mu \mathrm{m}$, and the waveguide spacing is $1 \mu \mathrm{m}$. The $1 \times 8$ AWG with 30 arrayed waveguides exhibits an insertion loss of $-3.4 \mathrm{~dB}$, a crosstalk of less than $-23.4 \mathrm{~dB}$, and a channel spacing of $1.9 \mathrm{~nm}$, as shown in Fig. 8(b)

\section{Interrogation Experimental Results and Discussion}

In the FBG sensor interrogation experiments, when the reflection light of FBG penetrates the AWG, light with a different wavelength separates and travels to different adjacent array waveguides. When each FBG reflection spectrum passes through the adjacent channel of the AWG, the reflection spectrum overlaps with the transmission spectrum of two AWG adjacent channels. $P_{i}$ and $P_{i+1}$ are the two adjacent channels of the output light intensity of AWG. Additionally, $P_{i}$ and $P_{i+1}$ are the convolutions of the FBG reflection spectrum and the AWG adjacent channel transmission spectrum, respectively. $P_{i}$ and $P_{i+1}$ are expressed as follows:

Table 1 Simulation results of the $1 \times 8$ AWG.

Type AWG core size $\left(\mu \mathrm{m}^{2}\right) \quad$ Insertion loss $(\mathrm{dB})$ Crosstalk (dB) Wavelength range $(\mathrm{nm}) \quad$ Channel spacing $(\mathrm{nm}) \quad$ Wavelength drift $(\mathrm{nm})$

\begin{tabular}{lllllll}
\hline $1 \times 8$ & $350 \times 250$ & -3.2 & -23.7 & 1543 to 1558 & 1.9 & 0.3 \\
\hline
\end{tabular}




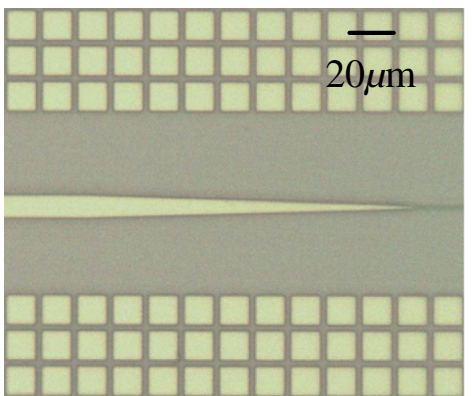

(a)

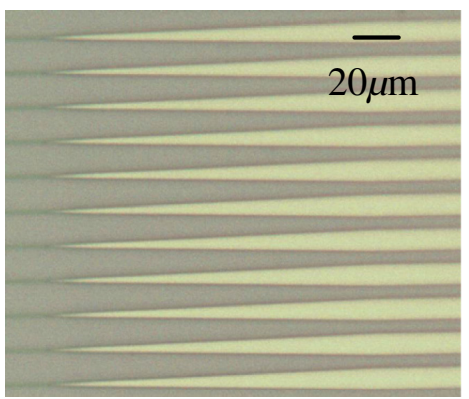

(b)

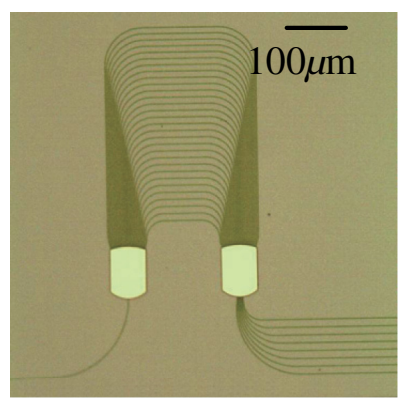

(c)

Fig. 7 Schematic layout of the microscopy images of the (a) input spot-size converter, (b) output spot-size converter, and (c) $1 \times 8$ AWG with 30 arrayed waveguides (core size is $350 \mu \mathrm{m} \times 250 \mu \mathrm{m}$ ).

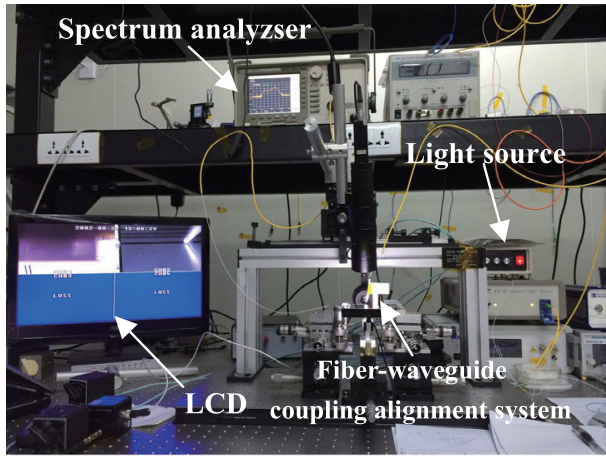

(a)

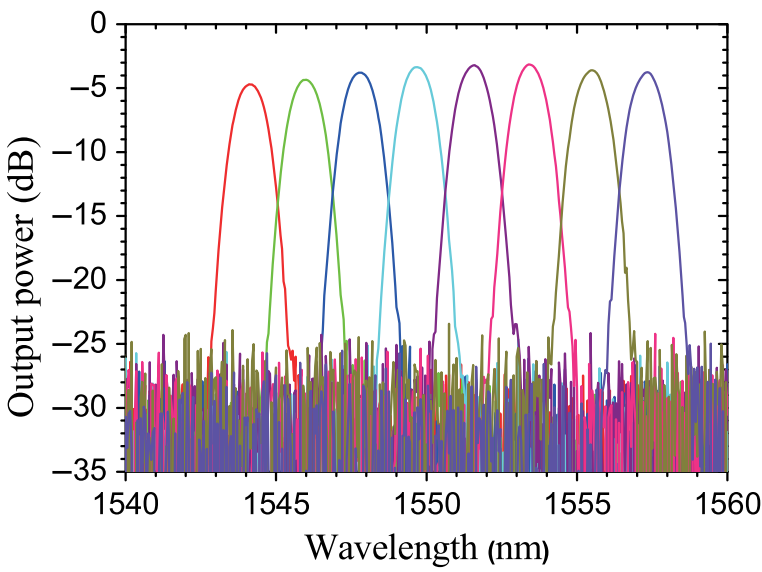

(b)

Fig. 8 Experimental results. (a) Optical experimental setup consisting of a light source, tapered lensed single-mode fibers, a fiber-waveguide coupling alignment system, and a spectrum analyzer. (b) Experimental results obtained with the $1 \times 8$ AWG with 30 arrayed waveguides.

$$
\begin{aligned}
& P_{i}=\left(1-L_{i}\right) \int_{0}^{\infty} S(\lambda) \cdot R_{\mathrm{FBG}}(\lambda) \cdot T_{\mathrm{AWG}}(i, \lambda) \mathrm{d} \lambda \\
& P_{i+1}=\left(1-L_{i+1}\right) \int_{0}^{\infty} S(\lambda) \cdot R_{\mathrm{FBG}}(\lambda) \cdot T_{\mathrm{AWG}}(i+1, \lambda) \mathrm{d} \lambda,
\end{aligned}
$$

where $S(\lambda)$ is the output power spectrum of the light source with the assumption that the output power is constant $S_{0}$ within the AWG bandwidth. $L_{i}$ and $L_{i+1}$ are the light attenuation coefficients. $R_{\mathrm{FBG}}(\lambda)$ is the reflection spectrum function of the sensory grating. $T_{\mathrm{AWG}}(i, \lambda)$ and $T_{\mathrm{AWG}}(i+1, \lambda)$ are the AWG channels, and $i$ and $i+1$ are the transmission spectrum functions in the fiber grating interrogation system. The relationship between the adjacent channel light intensity ratio logarithm of AWG and that of the FBG central wavelength is written as follows:

$$
\ln \left(\frac{P_{i+1}}{P_{i}}\right)=\frac{8(\ln 2) \Delta \lambda}{\Delta \lambda_{i}^{2}+\Delta \lambda_{\mathrm{FBG}}^{2}} \lambda_{\mathrm{FBG}}-\frac{4(\ln 2)\left(\lambda_{i+1}^{2}-\lambda_{i}^{2}\right)}{\Delta \lambda_{i}^{2}+\Delta \lambda_{\mathrm{FBG}}^{2}},
$$

where $\Delta \lambda$ is the adjacent two-channel central wavelength difference in AWG at the initial temperature. When FBG is subjected to constant stress, $\lambda_{\text {FBG }}$ exhibits a linear relationship with temperature. Temperature change can be measured in real time by measuring the dual-channel light intensity and by calculating the logarithm of light intensity ratio.

In the FBG sensor interrogation experiment involving the $1 \times 8$ AWG, the central wavelengths of the FBG sensors are $1544.75,1549.05,1552.25$, and $1556.35 \mathrm{~nm}$. The bandwidths are less than $0.25 \mathrm{~nm}$. These experimental results indicate a Bragg wavelength shift of $\sim 10 \mathrm{pm} /{ }^{\circ} \mathrm{C}$ against temperature change over the tested wavelength range. The wavelength spacing of the AWG is less than $1.9 \mathrm{~nm}$, indicating that the range of the Bragg wavelength shift cannot exceed $1.9 \mathrm{~nm}$. The wavelength drift ranges of the four AWGs are less than $0.8 \mathrm{~nm}$, indicating that the measurable range is less than $80^{\circ} \mathrm{C}$, as shown in Fig. 9(a). However, the measurement range of the FBG sensor interrogation system is limited because of some reasons. In the temperature interrogation experiment, FBG sensors are placed on a temperature control platform. The actual temperature is the temperature of the temperature control platform, and the measured temperature is the temperature measured by the FBG sensor interrogation system. The experimental results show that the FBG sensor interrogation system displays high accuracy at $10^{\circ} \mathrm{C}$ to $50^{\circ} \mathrm{C}$. Figure 9(b) shows the wavelength drift of the four FBGs at different temperatures. Figure 9(c) shows the relationship between the measured 


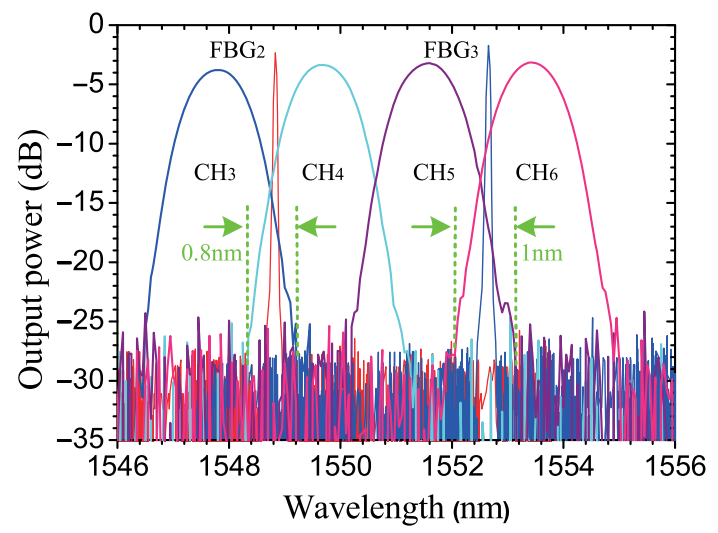

(a)

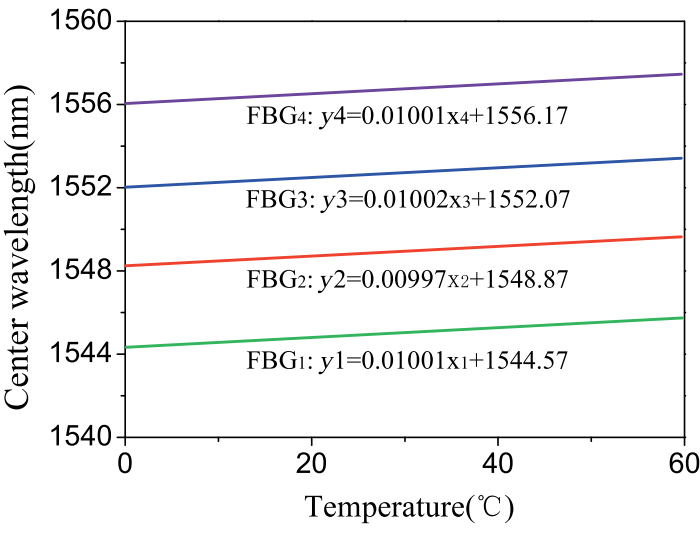

(b)

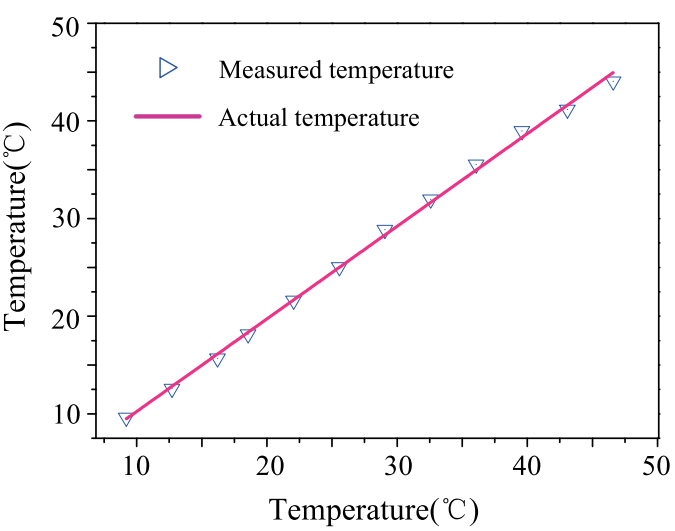

(c)

Fig. 9 (a) Reflected wave of FBG in different channels of $1 \times 8$ AWG with 30 arrayed waveguides $\left(\mathrm{CH}_{3}-\mathrm{CH}_{6}\right)$. (b) Wavelength drift of the four FBGs at different temperatures. (c) Relationship between the measured and actual temperatures.

Table 2 Experimental results of the $1 \times 8$ AWG.

\begin{tabular}{ccccc} 
Type & $\begin{array}{l}\text { AWG core } \\
\text { size }\left(\mu \mathrm{m}^{2}\right)\end{array}$ & $\begin{array}{c}\text { Insertion } \\
\text { loss }(\mathrm{dB})\end{array}$ & $\begin{array}{c}\text { Crosstalk } \\
(\mathrm{dB})\end{array}$ & $\begin{array}{c}\text { Channel } \\
\text { width }(\mathrm{nm})\end{array}$ \\
\hline $1 \times 8$ & $350 \times 250$ & -3.4 & -23.4 & 2.8 \\
\hline
\end{tabular}

and actual temperatures of one FBG sensor. The errors for all measured and actual temperatures are less than $0.3^{\circ} \mathrm{C}$ (Table 2).

The optimization scheme for the FBG sensor system has two directions. The first direction involves the optimization of device performance to improve system performance. Future work will focus on improving device performance to reduce insertion loss and channel crosstalk. The other scheme is the optimization of the demodulation algorithm used in tracking wavelength shifts in FBGs. The optimized algorithm should be more accurate and faster than other well-known demodulation algorithms.

\section{Conclusion}

An SoI-based AWG is proposed in this study. The core sizes of the proposed AWG are smaller than $530 \mu \mathrm{m} \times 480 \mu \mathrm{m}$. The simulation results show that the wavelength drifts of the $1 \times 8$ AWG are $0.3 \mathrm{~nm}$ under TE and TM modes. The polarization sensitivity of the AWG increases because of the increase in channels and tight structure. The experimental results of the optical elements show that the $1 \times 8$ AWG provides an insertion loss of less than $-3.4 \mathrm{~dB}$, a crosstalk of less than $-23.4 \mathrm{~dB}$, and a channel spacing of $1.9 \mathrm{~nm}$. The results of the temperature interrogation experiment show that the FBG sensor interrogation system based on the $1 \times 8$ AWG displays high interrogation accuracy at $10^{\circ} \mathrm{C}$ to $50^{\circ} \mathrm{C}$. Errors in the measured and actual temperatures are lower than $0.3^{\circ} \mathrm{C}$. The results of this study, which investigates the polarization sensitivity of ultrasmall $1 \times N$ SoI AWG, will serve as important references for future works on FBG sensor interrogation.

\section{Acknowledgments}

This work was supported by the National Natural Science Foundation of China (Grant Nos. 61711530652, 61675154, 61504093, and 61177078) and Sêr Cymru National Research Network in Advanced Engineering and Materials.

\section{References}

1. G. Rodriguez et al., "Coherent pulse interrogation system for fiber Bragg grating sensing of strain and pressure in dynamic extremes of materials," Opt. Express 23(11), 14219-14233 (2015).

2. G. Woyessa et al., "Temperature insensitive hysteresis free highly sensitive polymer optical fiber Bragg grating humidity sensor," Opt. Express 24(2), 1206-1213 (2016).

3. S. Kim, "Effect of sensing distance of aluminum-coated FBG sensors installed on a composite plate under a low-velocity impact," Compos. Struct. 160, 248-255 (2017). 
4. S. Zheng et al., "Sensitivity characterization of cladding modes in longperiod gratings photonic crystal fiber for structural health monitoring," Measurement 72, 43-51 (2015).

5. S. Zheng, Y. Zhu, and S. Krishnaswamy, "Fiber humidity sensors with high sensitivity and selectivity based on interior nanofilm-coated photonic crystal fiber long-period gratings," Sens. Actuators B 176. 264-274 (2013)

6. S. Zheng, M. Ghandehar, and J. Ou, "Photonic crystal fiber long-period grating absorption gas sensor based on a tunable erbium-doped fiber ring laser," Sens. Actuators B 223, 324-332 (2016).

7. S. Zheng, "Long-period fiber grating moisture sensor with nano-structured coatings for structural health monitoring," Struct. Health Monit. 14(2), 148-157 (2015).

8. J. Pan et al., "Optimization of dynamic matched grating filtering demodulation drived by piezoelectric ceramic," Acta Photonica Sin. 39(2), 243-246 (2010).

9. H. Gao et al., "InGaAs spectrometer and F-P filter combined FBG sensing multiplexing technique," T. Lightwave Technol. 26(19), 2282-2285 (2010).

10. W. Huang et al., "A cross-correlation method in wavelet domain for demodulation of FBG-FP static-strain sensors," EEEE Photonics Technol. Lett. 26(16), 1597-1600 (2014).

11. Y. Jiang et al., "Phase-shifted white-light interferometry for the absolute measurement of fiber optic Mach-Zehnder interferometers," J. Lightwave Technol. 28(22), 3294-3299 (2010).

12. J. Zhou et al., "Radio-frequency unbalanced M-Z interferometer for wavelength interrogation of fiber Bragg grating sensors," Opt. Lett. 41(2), 313-316 (2016).

13. L. S. Yan et al., "A simple demodulation method for FBG temperature sensors using a narrow band wavelength tunable DFB laser," IEEE Photonics Technol. Lett. 22(18), 1391-1393 (2010).

14. J. Koch, M. Angelmahr, and W. Schade, "Arrayed waveguide grating interrogator for fiber Bragg grating sensors: measurement and simulation," Appl. Opt. 51(31), 7718-7723 (2012).

15. B. Shen et al., "An integrated-nanophotonics polarization beamsplitter with $2.4 \times 2.4 \mu \mathrm{m}_{2}$ footprint," Nat. Photonics 9, 378-382 (2015).
16. S. Pathak et al., "Compact SOI-based AWG with flattened spectral response using MMI," in 8th IEEE Int. Conf. on Group IV Photonics, Vol. 8, pp. 45-47 (2011).

17. B. Yang et al., "Compact arrayed waveguide grating devices based on small SU-8 strip waveguides," t. Lightwave Technol. 29(13), 2009-2014 (2011)

18. J. Wang et al., "Low-loss and low-crosstalk $8 \times 8$ silicon nanowire AWG routers fabricated with CMOS technology," Opt. Express 22(8), 9395-9403 (2014).

19. J. Park et al., "Performance improvement in silicon arrayed waveguide grating by suppression of scattering near the boundary of a star coupler," Appl. Opt. 54(17), 5597-5602 (2015).

20. J. Zou et al., "Performance improvement for silicon-based arrayed waveguide grating router," Opt. Express 25(9), 9963-9973 (2017).

21. H. Li et al., "Design optimization and comparative analysis of siliconnanowire-based couplers," IEEE Photonics J. 4(5), 2017-2026 (2012).

22. H. Li et al., "Design of $1 \times 8 \mathrm{Si}$ nanowire AWG for on-chip AWG demodulation integration microsystem," Opt. Eng. 51(12), 123001 (2012).

23. $\mathrm{H}$. $\mathrm{Li}$ et al., "Investigation of the chip to photodetector coupler with subwavelength grating on SOI," Opt. Laser Technol. 76, 79-84 (2016)

Hongqiang Li is currently a professor in School of Electronics and Information Engineering at Tianjin Polytechnic University, China, and performs research activity at Tianjin Key Laboratory of Optoelectronic Detection Technology and Systems, China. He has published more than 130 journal and conference papers. His current research interests include fiber Bragg grating sensors, interrogation system, and silicon-based photonic integration.

Biographies for the other authors are not available. 